\title{
The optimal timing and management of hepatitis B and $C$ in patients with hepatocellular carcinoma
}

\author{
Thuy-Van P. Hang, Joel P. Wedd \\ Division of Digestive Diseases, Department of Medicine, Emory University School of Medicine, Atlanta, GA, USA \\ Contributions: (I) Conception and design: All authors; (II) Administrative support: JP Wedd; (III) Provision of study materials or patients: None; (IV) \\ Collection and assembly of data: None; (V) Data analysis and interpretation: None; (VI) Manuscript writing: All authors; (VII) Final approval of \\ manuscript: All authors. \\ Correspondence to: Joel P. Wedd, MD, MPH. Assistant Professor, Division of Digestive Diseases, Department of Medicine, Emory Transplant Center, \\ Emory University School of Medicine, 1365 Clifton Road, NE, Building A - Suite 1500, Atlanta, GA 30322, USA. Email: joel.wedd@emory.edu.
}

\begin{abstract}
Hepatocellular carcinoma (HCC) is the fourth most common cause of cancer related mortality worldwide, with the most common underlying etiologies being chronic hepatitis $\mathrm{B}$ and hepatitis $\mathrm{C}$ infections. Treatment of these viral hepatidities in the setting of HCC has been debated, and there is increasing study addressing this topic. Patients with advanced HCC of either etiology are unlikely to benefit from antiviral treatments, and futility should be considered prior to starting antiviral therapy. Hepatitis B treatment has demonstrated improved survival, decreased risk of hepatitis B reactivation, and decreased risk of late HCC recurrence. The mainstay treatment of chronic hepatitis B has been nucleos(t)ide analogues (NAs), and in the setting of HCC, entecavir and tenofovir are preferred given their higher potency and barriers to resistance. Those who were already on a NAs at the time of HCC diagnosis should be continued on them regardless of the HCC management planned. Patients who are suitable candidates to start NAs should start them at the time of HCC diagnosis. Direct-acting antivirals (DAAs) are the first line therapies for hepatitis C. Unlike with hepatitis B, those with $\mathrm{HCV}$-associated HCC are recommended to start treatment 3-6 months after complete treatment of their HCC, given lower rates of sustained virologic response (SVR) with active HCC. There are also controversial concerns about DAAs contributing to a more aggressive HCC phenotype, but data are limited by retrospective studies, and more recent retrospective studies are more reassuring. In transplant candidates, starting DAAs may be deferred until after transplant depending on median regional wait times, availability of $\mathrm{HCV}$ positive organs, and the degree of the patient's liver dysfunction. Overall, in patients with HCC from hepatitis B or C, treatment of the underlying viral hepatitis should be considered unless advanced stage limits benefits and results in futility.
\end{abstract}

Keywords: Hepatitis B therapy; hepatitis C therapy; hepatocellular carcinoma (HCC)

Submitted Jan 30, 2020. Accepted for publication May 14, 2020.

doi: $10.21037 / \mathrm{cco}-20-46$

View this article at: http://dx.doi.org/10.21037/cco-20-46

\section{Introduction}

Hepatocellular carcinoma (HCC), the most common type of primary liver cancer, has become the fourth leading cause of cancer-related mortality worldwide (1). HCC incidence rates have been increasing over the last thirty years, and these trends are expected to continue through 2030 (2), in part secondary to population growth and aging despite improvements in HCV therapy, making it all the more important to address any underlying risk factors. Chronic hepatitis $B$ virus (HBV) and chronic hepatitis $C$ virus (HCV) infections are prominent drivers of the development of cirrhosis and HCC around the world. While timely treatment of viral hepatitis has been shown to prevent the progression of cirrhosis and development of HCC, the decision to proceed with antiviral therapy after HCC diagnosis is more complicated. 


\section{Hepatitis B-related HCC}

\section{Chronic hepatitis B remains prevalent worldwide}

Around the world, more than 250 million people are infected with chronic HBV, most of whom are in the Western Pacific, Africa, and East Mediterranean (3). A majority of cases are acquired vertically from mother to child. Although effective vaccination programs around the world have helped decrease the incidence of new HBV infections, HBV remains an important cause of morbidity and mortality with regards to liver disease.

\section{Hepatitis B infection increases HCC risk}

Active viral replication of the $\mathrm{HBV}$ is associated with increased liver inflammation resulting in a risk of hepatocellular cancer even in the absence of underlying cirrhosis. This is thought to be due to the HBV deoxyribonucleic acid (DNA)'s integration into the host genome, which leads to genetic damage and malignant transformation $(4,5)$. Metabolic syndrome, central obesity, and type 2 diabetes have also been independently associated with increased HCC risk in HBV-infected patients in China (6). Persistent HBV e antigen and high HBV DNA, both of which suggest active viral replication, are associated with higher likelihood of developing HCC; in addition, heavy smoking and alcohol use in a person with $\mathrm{HBV}$ increases his or her risk of hepatocellular cancer by nine-fold (4).

\section{Start entecavir or tenofovir in hepatitis B-associated HCC}

Even in the case of HCC, viral inhibition of HBV can help preserve or improve liver function, increasing the patient's chances of getting therapeutic intervention for their HCC (5). Although historically, interferon- $\alpha$ played a role in the treatment of chronic HBV, it is not used in the settings of cirrhosis or HCC given the risk of decompensation (5). Currently, studies support nucleos(t)ide analogue (NA) therapy, which is well tolerated and has a good safety profile, in patients with HBV-related HCC. In the case of HCC, treatment of HBV is typically life-long and as such, only NAs with high potency and higher barriers to resistance such as entecavir, tenofovir disoproxil fumarate, and tenofovir alafenamide are recommended (5). Kim et al. have demonstrated that in such patients, entecavir has demonstrated overall improved survival, decompensationfree survival, and recurrence-free survival compared to lamivudine (7).
If a patient is already on an NA at the time of their diagnosis of HCC, it is usually continued irrespective of the planned HCC management (5). If they are not on treatment yet, the decision to start an NA is based on the treatability of their HCC and overall prognosis since the purpose of treating $\mathrm{HBV}$ in these situations is to preserve liver function and prevent de novo or recurrent HCCs (5). The role of starting NAs for incurable, advanced HCC is not well defined, and their use in these situations may be futile. However, if the patient is already taking a NA, they should continue it, given risk of flare with stopping treatment (5).

For patients on the waiting list for liver transplant with HBV-related HCC, NAs should be started at the time of HCC diagnosis, ideally one to three months prior to transplant, and continued lifelong after transplant in order to prevent graft hepatitis and graft loss $(5,8)$. Although liver transplant is curative for cirrhosis and HCC, it does not eradicate $\mathrm{HBV}$.

\section{HBV treatment in HCC improves survival}

Antivirals have demonstrated survival benefit in those with $\mathrm{HBV}$-associated hepatocellular cancer. A meta-analysis by Yuan et al. with 15 studies with 8,060 HBV-related HCC patients (2,499 treated vs. 5,562 untreated) demonstrated that overall survival was higher in those treated with antivirals by $11 \%$ at 1 year, $28 \%$ at 3 years, and $40 \%$ at 5 years, and disease free survival was greater in the treatment group by $17 \%(\mathrm{P}<0.008)(9)$. Similarly, a metaanalysis by Zhang et al. of 7,619 postoperative HBV-related HCC patients showed that antiviral therapy improves 1-, 3-, and 5 -year recurrence free survival and decreased mortality after curative resection compared to controls (10).

In a randomized control trial by Yin et al., post hepatectomy treatment with NAs demonstrated decreased HCC recurrence (HR 0.48, 95\% CI: 0.32-0.70) and reduced HCC related death (HR 0.26, 95\% CI: 0.14-0.50) compared to no HBV treatment (11). High viral load (HBV DNA $\geq 10^{4}$ copies $/ \mathrm{mL}$ ) was associated with unfavorable overall survival and relapse free survival after hepatectomy, while the use of antivirals was associated with improved overall survival and relapse free survival (11). Antivirals were associated with decreased early recurrence compared to controls (HR $=0.41)(11)$.

\section{$N A$ s reduce $H B V$ reactivation}

Treatment modalities for HCC such as chemotherapy, 
surgery, or associated anesthesia, can lead to impaired host immunity and a relative immunosuppressed state, which in turn can prompt HBV reactivation. Reactivation is defined as a ten-fold increase in HBV DNA compared to baseline, reappearance of hepatitis B surface antigen, or serum HBV DNA $>200 \mathrm{IU} / \mathrm{mL}$ in baseline hepatitis B surface antigen negative patients (8). HBV reactivation after HCC treatment can lead to poor outcomes. Hepatitis B e antigen positivity, detectable preoperative HBV DNA, high Ishak inflammatory score, preoperative transarterial chemoembolization (TACE), longer operating room time, and blood transfusion were independent risk factors for reactivation of hepatitis B after surgery for HCC (12). A study conducted by Huang et al. found that patients who had HBV reactivation postoperatively had high rates of mortality from liver failure compared to those who did not (11.8\% vs. 6.4\%, respectively, $\mathrm{P}=0.002)$ (12). At three years, the disease-free survival of those with $\mathrm{HBV}$ reactivation was $34.1 \%$ compared to $46.0 \%$ in those without reactivation $(\mathrm{P}=0.009)$, and the overall survival was significantly lower in those with reactivation compared to those without $(51.6 \%$ vs. $67.2 \%$, respectively, $\mathrm{P}<0.001)(12)$. The use of NAs prior to HCC treatment can help preserve or improve liver function, preventing ongoing inflammation and subsequent HBV reactivation (8).

The rate of $\mathrm{HBV}$ reactivation in the group with negative HBV DNA preoperatively was significantly lower than those who had detectable HBV DNA preoperatively $(16.7 \%$ vs. $29.4 \%$, respectively, $\mathrm{P}<0.001)$ (12). Even low preoperative viral loads (HBV DNA $<2,000 \mathrm{IU} / \mathrm{mL}$ ) showed an increased risk of $\mathrm{HBV}$ reactivation, demonstrating the importance of prophylactic antivirals. Multiple studies have demonstrated that after treatment with resection or locoregional therapy (LRT), HBV reactivation rates, liver dysfunction, and rate of de novo HCC in the remnant liver are lower in those on antivirals compared to those who are not $(5,10,13)$. As such, NAs should be started prior to curative HCC treatment (i.e., resection and TACE) in those with hepatitis B surface antigen positivity, even if HBV DNA is negative, and in those with detectable HBV DNA, even at low levels $(8,10)$.

\section{HBV treatment can lower HCC recurrence}

In those with HBV-related HCC treated with antivirals, HCC recurrence was significantly decreased at one year if treated (RR 0.41, 95\% CI: $0.28-0.61, \mathrm{P}<0.00001$ ), as well as at three year follow up (RR 0.63, 95\% CI: 0.43-0.94, $\mathrm{P}=0.001)(9)$. Reappearance of hepatitis B surface antigen and HBV DNA after transplant is highly associated with HCC recurrence (14). A case control study of 399 patients with HBV-related HCC after radiofrequency ablation (RFA) showed that antivirals were independently associated with decreased risk of HCC recurrence (HR 0.69, 95\% CI: 0.50-0.95, $\mathrm{P}<0.05)(15)$. Fung and Chok, however, found that while the treatment of $\mathrm{HBV}$ with NAs has shown to prevent reactivation and graft hepatitis after liver transplant, it is unlikely to prevent early HCC recurrence, which is attributed to intrahepatic metastatic disease (5).

In summary, the use of NAs to lower HBV DNA levels in those with hepatocellular cancer, has been shown to improve overall survival, recurrence free survival, HBV reactivation, and late HCC recurrence.

\section{Hepatitis C-related HCC}

\section{Chronic HCV is prevalent in Western countries}

Around the world, approximately 71 million people are living with hepatitis $\mathrm{C}$, with the highest incidences in the Eastern Mediterranean and European regions (3). Unlike hepatitis B infections, the predominant modes of transmission of the HCV are from unsafe health care injections and recreational injection drug use. In the United States, about 3.5 million people carry the $\mathrm{HCV}$, with more than $75 \%$ of them from the "baby boomer" age cohort (born between 1945 and 1965) (3).

\section{Hepatitis C increases HCC risk}

Currently, hepatitis C is the most common cause of HCC in Western countries, typically in the setting of advanced fibrosis or cirrhosis. There is an annual $2-4 \%$ risk of developing HCC in those with chronic HCV and cirrhosis (16). Treatment with direct-acting antivirals (DAAs) has been associated with a relative risk reduction of HCC incidence in both those with and without cirrhosis (16). Patients with $\mathrm{HCV}$ cirrhosis unfortunately have ongoing risk of HCC even after treatment of their hepatitis $\mathrm{C}$, albeit less than if they remained untreated. Studies have shown that HCC can develop even 10 years after sustained virologic response (SVR) (4).

\section{Conflicting data in treating HCV in HCC patients}

In the past, the mainstay treatment of hepatitis $\mathrm{C}$ involved interferon-based antiviral treatment (17-19). A meta- 
analysis by Manthravadi et al. demonstrated that SVR with interferon was associated with improved survival and progression-free survival in HCC (18-20). Now, welltolerated combinations of DAAs are the first line therapies, with SVR rates exceeding 95\% $(4,21)$.

Recent retrospective studies regarding the use of DAAs for HCV treatment in those with HCC were concerning for increased incidence of recurrent HCC after successful DAA $(22,23)$. However, larger studies, albeit still retrospective, are more reassuring $(24,25)$. In a systematic review of 41 studies with 13,875 patients with $\mathrm{HCV}$ treated with interferon or DAA, there was no difference in HCC recurrence risk after SVR from DAA compared to interferon-based treatment (25). A large, multicenter retrospective cohort study by Singal et al. involving 797 patients from the United States and Canada showed no difference in overall or early HCC recurrence between those who took DAAs after complete HCC response and those who did not take DAAs (26). Multivariable models demonstrated that DAAs were not associated with HCC recurrence (HR 0.980, 95\% CI: $0.7-1.16)$ and that they were associated with lower mortality (HR 0.54, 95\% CI: 0.33-0.90) (26). Those who obtained sustained virologic response on DAA had a significantly reduced mortality compared to those who did not obtain SVR (HR 0.27, 95\% CI: 0.13-0.57) (26). There was no morality benefit between those who were on DAA and did not obtain SVR compared to untreated patients (HR 1.13, 95\% CI: 0.55-2.33) (26). While the recent studies are reassuring, the concern remains controversial and larger prospective studies are needed to resolve the debate. It is also important to consider that the use of DAAs can help improve portal hypertension and liver dysfunction, two major causes of death in those who have treated HCC and untreated hepatitis $\mathrm{C}(16)$.

\section{Active HCC decreases the rate of sustained virologic response}

While DAAs are highly successful in inducing SVR, this appears tempered in the setting of active hepatocellular cancer. In a single center study by Prenner et al. with 421 patients with $\mathrm{HCV}$ cirrhosis on DAA, $21 \%$ of patients with HCC were unable to achieve sustained virologic response compared to $12 \%$ of patients who did not have underlying HCC $(\mathrm{P}=0.009)$ (27). Those who had inactive HCC or were treated with DAA after HCC resection or liver transplant had SVR rates similar to those who did not have HCC $(\mathrm{P}<0.0001)(27)$. Active HCC at the time of HCV treatment initiation was significantly associated with DAA treatment failure (adjusted OR 8.5, 95\% CI: 3.90-18.49) (27). A study completed by Beste $e t$ al. involving patients in the national Veterans Affairs health care system similarly found that HCC was associated with lower SVR rates with DAAs (adjusted OR 0.38, 95\% CI: 0.29-0.48, $\mathrm{P}<0.001$ ), but that $\mathrm{HCV}$ can be cured in a majority of patients who had prior HCC, including those who underwent liver transplant (28). Those without HCC had $91.1 \%$ SVR compared to $74.4 \%$ in those with HCC, and there was $94.0 \%$ SVR in those who had HCC and subsequently underwent liver transplant (28). Another systemic review and meta-analysis of 49 studies with 39,042 patients showed a pooled SVR rate of $89.6 \%$ in those with HCC compared to $93.3 \%$ in those without $(\mathrm{P}=0.0012)$, supporting the previous data that those with active or residual HCC are less likely to obtain SVR than their counterparts (29).

\section{Delay hepatitis treatment until after HCC treatment}

The data available thus far suggest that deferring DAA therapy until after HCC treatment in those eligible for potentially curative HCC therapies such as resection or LRT is the optimal strategy in eradicating HCV in this population. Per the American Association for the Study of Liver Diseases' practice guidance and American Gastroenterology Association Clinical Practice Update, it is recommended to treat hepatitis $\mathrm{C}$ infection after HCC is completely treated without any evidence of recurrence after 3-6 months $(4,16,30)$. This delay in initiating HCV therapies provides enough time to confirm complete HCC treatment since early HCC recurrence can occur from intrahepatic metastases that were not previously detected, with the goal of avoiding HCC-related lower SVR rates.

In patients with active intermediate or advanced HCC, there is insufficient data regarding the benefits and costeffectiveness of treating $\mathrm{HCV}$, and $\mathrm{HCV}$ treatment is typically deferred in this population (16).

\section{DAAs in patients listed for liver transplant}

In those who are listed for liver transplant, the decision for timing of DAAs should be made with regards to median regional wait times, availability of $\mathrm{HCV}$ positive organs, and the degree of liver dysfunction. In the United States, most HCV positive donor livers are primarily from the baby boomer cohort or injection drug users. With the opioid epidemic, not only is the prevalence of $\mathrm{HCV}$ 
infection increasing among this group, but also the deaths related to opioid overdose, and thus pool of $\mathrm{HCV}$ positive liver donors (31). However, in areas where there are long wait times or limited HCV positive donors, DAAs prior to transplant may be beneficial (16). A single-center study of 149 liver transplant candidates with HCV-related HCC showed that the risk of HCC recurrence in one year after complete response to LRT was not significantly different between those who were not treated compared to those who were treated with DAAs (adjusted HR 0.91, 95\% CI: $0.58-1.42)$ (32). In addition, those who were treated with DAAs had lower risk of waitlist dropout related to tumor progression or death compared to those who were untreated (adjusted HR 0.30, 95\% CI: 0.13-0.69) (32).

Waiting until after treatment of HCC before using DAAs appears to restore SVR rates, and those with SVR have decreased mortality. Whether there is an increased risk of HCC recurrence with the use of DAAs remains controversial with conflicting data, and prospective study could help resolve the concern.

\section{Conclusions}

HCC remains a prominent cause of cancer globally, with hepatitis $\mathrm{B}$ and hepatitis $\mathrm{C}$ being significant underlying risk factors. While treating $\mathrm{HBV}$ and $\mathrm{HCV}$ reduces the risk of progression to cirrhosis and HCC, the role of therapy in the setting of newly diagnosed HCC remains unclear. In general, those with advanced HCC of either etiology are less likely to benefit from starting antiviral therapies, and those who have already been on treatment for their viral hepatitis should continue it. In those who show favorable prognosis and have treatable HCC, there is a role for antivirals either at the time of HCC diagnosis for HBV patients or 3 to 6 months after treatment of HCC in the case of HCV-related HCC.

\section{Acknowledgments}

Funding: None.

\section{Footnote}

Provenance and Peer Review: This article was commissioned by the Guest Editors (Mehmet Akce and Shishir K. Maithel) for the series "Hepatocellular Carcinoma" published in Chinese Clinical Oncology. The article was sent for external peer review organized by the Guest Editors and the editorial office.

Conflicts of Interest: Both authors have completed the ICMJE uniform disclosure form (available at http://dx.doi. org/10.21037/cco-20-46). The series "Hepatocellular Carcinoma" was commissioned by the editorial office without any funding or sponsorship. The authors have no other conflicts of interest to declare.

Etbical Statement: The authors are accountable for all aspects of the work in ensuring that questions related to the accuracy or integrity of any part of the work are appropriately investigated and resolved.

Open Access Statement: This is an Open Access article distributed in accordance with the Creative Commons Attribution-NonCommercial-NoDerivs 4.0 International License (CC BY-NC-ND 4.0), which permits the noncommercial replication and distribution of the article with the strict proviso that no changes or edits are made and the original work is properly cited (including links to both the formal publication through the relevant DOI and the license). See: https://creativecommons.org/licenses/by-nc-nd/4.0/.

\section{References}

1. Yang JD, Hainaut P, Gores GJ, et al. A global view of hepatocellular carcinoma: trends, risk, prevention and management. Nat Rev Gastroenterol Hepatol 2019;16:589-604.

2. Petrick JL, Kelly SP, Altekruse SF, et al. Future of hepatocellular carcinoma incidence in the united states forecast through 2030. J Clin Oncol 2016;34:1787-94.

3. Mak LY, Cruz-Ramon V, Chinchilla-Lopez P, et al. Global Epidemiology, Prevention, and Management of Hepatocellular Carcinoma. Am Soc Clin Oncol Educ Book 2018;38:262-79.

4. Marrero JA, Kulik LM, Sirlin CB, et al. Diagnosis, Staging, and Management of Hepatocellular Carcinoma: 2018 Practice Guidance by the American Association for the Study of Liver Diseases. Hepatology 2018;68:723-50.

5. Fung J, Chok KSH. The role of oral antiviral therapy in hepatitis B-related hepatocellular carcinoma. Hepatoma Res 2017;3:284-93.

6. Tan Y, Zhang X, Zhang W, et al. The Influence of Metabolic Syndrome on the Risk of Hepatocellular Carcinoma in Patients with Chronic Hepatitis B Infection in Mainland China. Cancer Epidemiol Biomarkers Prev 
2019;28:2038-46.

7. Kim JH, Sinn DH, Kim K, et al. Lamivudine versus entecavir for newly diagnosed hepatitis B virus-related hepatocellular carcinoma. Gut Liver 2016;10:939-47.

8. Zhang YQ, Guo JS. Antiviral therapies for hepatitis B virus-related hepatocellular carcinoma. World J Gastroenterol 2015;21:3860-6.

9. Yuan P, Chen P, Qian Y. Evaluation of Antiviral Therapy Performed after Curative Therapy in Patients with HBVRelated Hepatocellular Carcinoma: An Updated MetaAnalysis. Can J Gastroenterol Hepatol 2016;2016:5234969.

10. Zhang H, Zhou Y, Yuan G, et al. Antiviral therapy improves the survival rate and decreases recurrences and fatalities in liver cancer patients following curative resection: a metaanalysis. Mol Clin Oncol 2015;3:1239-47.

11. Yin J, Li N, Han Y, et al. Effect of antiviral treatment with nucleotide/nucleoside analogs on postoperative prognosis of hepatitis B virus-related hepatocellular carcinoma: a two-stage longitudinal clinical study. J Clin Oncol 2013;31:3647-55.

12. Huang G, Lai EC, Lau WY, et al. Posthepatectomy $\mathrm{HBV}$ reactivation in hepatitis $\mathrm{B}$-related hepatocellular carcinoma influences postoperative survival in patients with preoperative low HBV-DNA levels. Ann Surg 2013;257:490-505.

13. Lao XM, Luo G, Ye LT, et al. Effects of antiviral therapy on hepatitis $\mathrm{B}$ virus reactivation and liver function after resection or chemoembolization for hepatocellular carcinoma. Liver Int 2013;33:595-604.

14. Campsen J, Zimmerman M, Trotter J, et al. Liver transplantation for hepatitis B liver disease and concomitant hepatocellular carcinoma in the United States with hepatitis B immunoglobulin and nucleoside/ nucleotide analogues. Liver Transpl 2013;19:1020-9.

15. Lee TY, Lin JT, Zeng YS, et al. Association between nucleos(t)ide analog and tumor recurrence in hepatitis $\mathrm{B}$ virus-related hepatocellular carcinoma after radiofrequency ablation. Hepatology 2016;63:1517-27.

16. Singal AG, Lim JK, Kanwal F. AGA Clinical Practice Update on Interaction Between Oral DirectActing Antivirals for Chronic Hepatitis C Infection and Hepatocellular Carcinoma: Expert Review. Gastroenterology 2019;156:2149-57.

17. Cardoso AC, Moucari R, Figueiredo-Mendes C, et al. Impact of peginterferon and ribavirin therapy on hepatocellular carcinoma: incidence and survival in hepatitis $\mathrm{C}$ patients with advanced fibrosis. J Hepatol 2010;52:652-7.
18. Breitenstein S, Dimitroulis D, Petrowsky H. Systematic review and meta-analysis of interferon after curative treatment of hepatocellular carcinoma in patients with viral hepatitis. Br J Surg 2009;96:975-81.

19. Knop V, Hoppe D, Welzel T, et al. Regression of fibrosis and portal hypertension in $\mathrm{HCV}$-associated cirrhosis and sustained virologic response after interferon-free antiviral therapy. J Viral Hepat 2016;23:994-1002.

20. Manthravadi S, Paleti S, and Pandya P. Impact of sustained viral response postcurative therapy of hepatitis C-related hepatocellular carcinoma: a systematic review and metaanalysis. Int J Cancer 2017;140:1042-9.

21. Falade-Nwulia O, Suarez-Cuervo C, Nelson DR, et al. Oral direct-acting agent therapy for hepatitis $\mathrm{C}$ virus infection. Ann Intern Med 2017;166:637-48.

22. Conti F, Buonfiglioli F, Scuteri A, et al. Early occurrence and recurrence of hepatocellular carcinoma in HCVrelated cirrhosis treated with direct-acting antivirals. J Hepatol 2016;65:727-33.

23. Minami T, Tateishi R, Nakagomi R, et al. The impact of direct-acting antivirals on early tumor recurrence after radiofrequency ablation in hepatitis C-related hepatocellular carcinoma. J Hepatol 2016;65:1272-3.

24. Ioannou GN, Green PK, Berry K. HCV eradication induced by direct-acting antiviral agents reduces the risk of hepatocellular carcinoma. J Hepatol 2017;68:25-32.

25. Waziry R, Hajarizadeh B, Grebely J, et al. Hepatocellular carcinoma risk following direct-acting antiviral $\mathrm{HCV}$ therapy: a systematic review, meta-analyses, and metaregression. J Hepatol 2017;67:1204-12.

26. Singal AG, Rich NE, Mehta N, et al. Direct-Acting Antiviral Therapy Not Associated With Recurrence of Hepatocellular Carcinoma in a Multicenter North American Cohort Study. Gastroenterology 2019;156:1683-1692.e1.

27. Prenner SB, VanWagner LB, Flamm SL, et al. Hepatocellular carcinoma decreases the chance of successful hepatitis $\mathrm{C}$ virus therapy with direct-acting antivirals. J Hepatol 2017;66:1173-81.

28. Beste LA, Green PK, Berry K, et al. Effectiveness of hepatitis $\mathrm{C}$ antiviral treatment in a USA cohort of Veteran patients with hepatocellular carcinoma. J Hepatol 2017;67:32-9.

29. Ji F, Yeo YH, Wei MT, et al. Sustained virologic response to direct-acting antiviral therapy in patients with chronic hepatitis $\mathrm{C}$ and hepatocellular carcinoma: A systematic review and meta-analysis. J Hepatol 2019;71:473-85.

30. Marrero JA, Singal AG. Direct-acting antivirals and recurrence of hepatocellular carcinoma. Liver Transpl 
2017;23:1099-100.

31. Crismale JF, Ahmad J. Expanding the donor pool: Hepatitis C, hepatitis B and human immunodeficiency virus-positive donors in liver transplantation. World J Gastroenterol 2019;25:6799-812.

Cite this article as: Hang TVP, Wedd JP. The optimal timing and management of hepatitis $\mathrm{B}$ and $\mathrm{C}$ in patients with hepatocellular carcinoma. Chin Clin Oncol 2021;10(1):4. doi: $10.21037 /$ cco-20-46
32. Huang AC, Mehta N, Dodge JL, et al. Direct-acting antivirals do not increase the risk of hepatocellular carcinoma recurrence after local-regional therapy or liver transplant waitlist dropout. Hepatology 2018;68:449-61. 